Europhys. Lett., 67 (1), pp. 42-48 (2004)

DOI: $10.1209 / \mathrm{epl} / \mathrm{i} 2003-10279-7$

\title{
Silica tubes in chemical gardens: Radius selection and its hydrodynamic origin
}

\author{
S. Thouvenel-Romans ${ }^{1}$, W. van SaArloos ${ }^{2}$ and O. $\operatorname{Steinbock}^{1}\left({ }^{*}\right)$ \\ 1 Department of Chemistry and Biochemistry, Florida State University \\ Tallahassee, FL 32306-4390, USA \\ 2 Instituut-Lorentz, Universiteit Leiden - Postbus 9506 \\ 2300 RA Leiden, The Netherlands
}

(received 17 October 2003; accepted in final form 3 May 2004)

PACS. 47.54.+r - Pattern selection; pattern formation.

PACS. 47.15. $-\mathrm{x}$ - Laminar flows.

PACS. 82.40.Ck - Pattern formation in reactions with diffusion, flow and heat transfer.

\begin{abstract}
Chemical gardens consist of hollow silica fibers that form from silicate solution upon seeding with salt crystals or injection of salt solution. We investigate the outer radius of these tubes for steady and oscillatory growth dynamics. The radius increases with increasing injection rates and concentrations of cupric sulfate seed solution. For steady growth, we find that the tube radii are described quantitatively by the Poiseuille-flow characteristics of the buoyant jet of injected solution. The oscillatory regime gives rise to wider tubes and involves the cyclic expansion and detachment of a membrane-bound droplet at the growth point. The droplets' expansion rate equals the applied injection rate indicating that, in this growth regime, the fluid flow is constrained to the interior of the silica structures.
\end{abstract}

A variety of seemingly unrelated reaction-precipitation systems can give rise to the growth of mesoscopic tubular structures. Examples include hollow rust fibers [1,2] formed in corrosion processes and microscopic, needle-like tubes created during the setting of cement [3]. The latter example is closely related to the formation of silica tubes in chemical gardens which are a well-known demonstration experiment in physics and chemistry [4]. Chemical gardens involve the growth of colorful, plant-like fibers from aqueous solutions containing anions such as aluminate, borate, carbonate, or silicate [5-7]. These structures have diameters in the micro- and millimeter range and reach lengths of several decimeters. Tube growth can be induced by seeding the latter solutions with crystals of various soluble salts (e.g., $\mathrm{CuSO}_{4}$, $\mathrm{MnCl}_{2}, \mathrm{FeCl}_{3}$ ) excluding group (I) compounds [7].

Early references to chemical gardens can be traced back to the 17 th century $[8,9]$. In the 20th century, these structures attracted considerable interest because they were mistaken to be prototype models of simple life forms [10]. Recently, the study of chemical gardens has been experiencing a renaissance as tubular structures in the aluminosilicate system have been shown to be promising catalytic materials with an intriguing hierarchical nanostructure [11,12]. For

(*) E-mail: steinbck@chem.fsu.edu

(c) EDP Sciences 

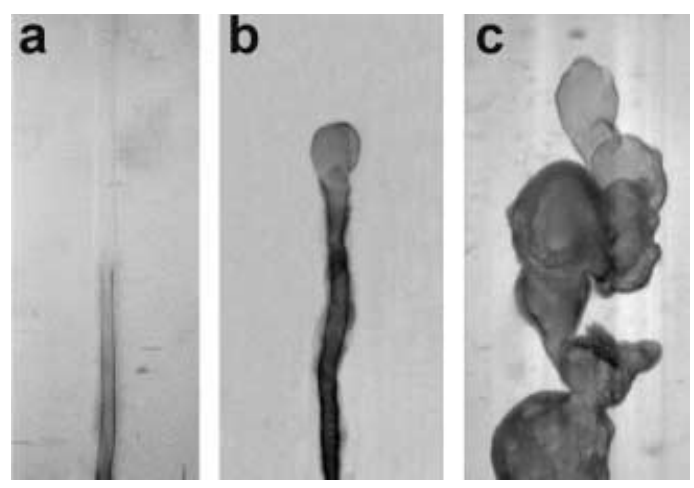

Fig. 1 - Representative micrographs of jetting (a), popping (b), and budding (c) silica tubes in the $\mathrm{CuSO}_{4} / \mathrm{Na}_{2} \mathrm{SiO}_{3}$ system. Concentration of the injected seed solution $\left[\mathrm{CuSO}_{4}\right]=0.05$ (a), 0.25 (b), and $0.50(\mathrm{c}) \mathrm{mol} / \mathrm{L}$. Flow rate $7.0 \mathrm{~mL} / \mathrm{h}(\mathrm{a}-\mathrm{c})$. Field of view: $2.6 \times 6.4(\mathrm{a})$ and $7.3 \times 15.0 \mathrm{~mm}^{2}(\mathrm{~b}, \mathrm{c})$.

example, Collins et al. [11] found that their overall amorphous samples involve $1 \mathrm{~nm}$ silica rods that self-organize into cylindrical clusters of about $40 \mathrm{~nm}$ within patterned micrometerscale domains.

The qualitative growth mechanism of chemical gardens is based on the formation of a semipermeable membrane around the dissolving seed crystal (see, e.g., ref. [5]). Osmotic pressure gives rise to the inflow of water causing the expansion and rupture of the membrane. Since the dissolution of the seed continues, osmotic pump action leads to the ejection of buoyant salt solution into the surrounding medium. In conjunction with polymerization and precipitation reactions [7], this process forms tubular structures that grow upward with speeds of $\mathrm{mm} /$ day to $\mathrm{mm} / \mathrm{s}$.

Major obstacles towards quantitative investigations of chemical gardens include the lack of appropriate control parameters and the transient character of the dissolution process. Recently, however, two of us [13] developed an alternative experimental approach that involves the hydrodynamic injection of "seed solution" into a large volume of silicate solution. By these means, three distinct growth regimes were identified for which examples are shown in fig. 1. For pump rates of $2-40 \mathrm{~mL} / \mathrm{h}$ and low cupric sulfate concentrations, one observes steady silica tube growth around a stable jet of seed solution (fig. 1a). At an increased seed concentration of approximately $0.1 \mathrm{~mol} / \mathrm{L}$, this jetting mode gives way to oscillatory dynamics that involve the rhythmic formation and release of a membrane-bound droplet at the growth point (fig. 1b). At $0.4 \mathrm{~mol} / \mathrm{L}$, this popping regime changes to budding growth in which the droplet is not released but bursts to nucleate a new, expanding bud at the breach site (fig. 1c). Moreover, it was shown that buoyant forces play a major role in the observed dynamics. Here, we present experimental data on the concentration and pump rate dependencies of the radius of silica tubes grown in the jetting and the popping regimes. These data are complemented by time-resolved measurements of the volume of popping tubes. In addition, a hydrodynamic description of the radius of jetting tubes is given.

Our experiments employ the injection technique first described in ref. [13]. A syringe pump (KD Scientific 200) is used to deliver cupric sulfate solution $\left(\left[\mathrm{CuSO}_{4}\right]<0.5 \mathrm{~mol} / \mathrm{L}\right.$, Fisher) into a large volume of waterglass $\left(\left[\mathrm{Na}_{2} \mathrm{SiO}_{3}\right]=1 \mathrm{~mol} / \mathrm{L}\right.$, Fluka). Injection is carried out in upward direction through a glass capillary (length $=4.9 \mathrm{~cm}$; inner diameter $\approx 0.8 \mathrm{~mm}$ ) at constant pump rate. A cylindrical glass vessel (height $\approx 30 \mathrm{~cm}$; inner diameter $=2.2 \mathrm{~cm}$ ) serves as the container for the silicate solution. All solutions are prepared in nano-pure 

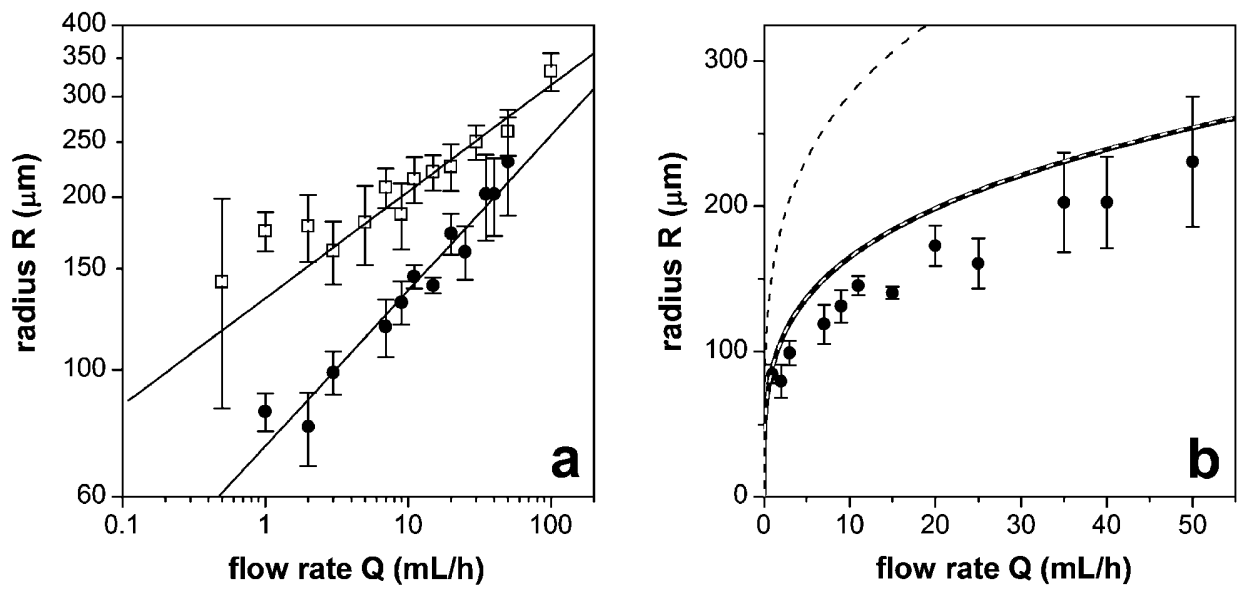

Fig. 2 - Flow rate dependence of the outer radius of silica tubes. Solid circles and open squares indicate seed concentration of $\left[\mathrm{CuSO}_{4}\right]=0.075$ and $0.15 \mathrm{~mol} / \mathrm{L}$, respectively. The lines in (a) are the results of linear regressions yielding power law exponents of 0.27 and 0.18 for jetting and popping growth, respectively. The jetting data are shown again in (b) along with the solutions of purely hydrodynamic equations. The solid curve and the nearly identical dashed line correspond to a model without adjustable parameters (cf. eqs. (1)) with the latter curve being the approximation specified in eq. (2). The upper dashed curve corresponds to the stiff tube model (i.e. eq. (2) for $\eta_{\mathrm{o}} \rightarrow \infty$ ).

water $(18 \mathrm{M} \Omega \mathrm{cm})$. Optical micrographs are acquired with a monochrome charged-coupled device camera (COHU 2122) connected to a PC via a frame grabber board (Data Translation, $640 \times 480$ pixel at $8 \mathrm{bit} / \mathrm{pixel}$ ). Image sequences are captured during the experiment using HLImage ++97 software and are analyzed with in-house-software. All experiments are carried out at $(25 \pm 1){ }^{\circ} \mathrm{C}$.

The data shown in fig. 2 describe the flow rate dependence of the outer radii of silica tubes in the $\mathrm{CuSO}_{4} / \mathrm{Na}_{2} \mathrm{SiO}_{3}$ system. The tube radii are measured from optical micrographs of numerous samples far away $(1-3 \mathrm{~cm})$ from the orifice of the glass capillary. Moreover, measurements are carried out at various positions along the individual tube's axis because most samples show erratic variations of their radius. These variations are reflected in the error bars that represent the standard deviations of the data obtained. The concentration of waterglass is kept constant at $1 \mathrm{~mol} / \mathrm{L}$ and the concentration of cupric sulfate is either $0.075 \mathrm{~mol} / \mathrm{L}$ (circles) or $0.15 \mathrm{~mol} / \mathrm{L}$ (squares). For all flow rates studied, the lower concentration gives rise to jetting growth whereas the higher value induces popping dynamics with average periods of $1-15 \mathrm{~s}$.

Our measurements reveal that the outer tube radius $R$ increases with increasing flow rates $Q$. Moreover, we find that, for any given flow rate, popping tubes are wider than those grown in the jetting regime (fig. 2a). The double-logarithmic plot suggests that the radii obey power-law-like dependencies. Assuming that $R \propto Q^{\alpha}$, linear regression yields exponents of $\alpha=0.27 \pm 0.02$ and $0.18 \pm 0.02$ for jetting and popping growth, respectively. We did not attempt to measure the radii of budding tubes (cf. fig. 1c) because their bulging shape does not allow accurate characterizations. Qualitatively, however, we find that budding tubes are significantly wider than those obtained under popping and jetting conditions.

We now show, quite remarkably, that in the jetting regime the growth of the silica tubes is driven by simple hydrodynamics, rather than by the properties of the membrane separating the silicate solution from the injected fluid. We start by assuming that the membrane is 
thin and mechanically weak. Based on this assumption, the shear stress and the velocity difference across the membrane can be neglected. For calculating the hydrodynamic flow, we can therefore focus on the fluid motion inside and outside the tube.

The outer silicate solution is confined to a glass cylinder of radius $R_{\text {cyl }}$ (here $11 \mathrm{~mm}$ ). Along its central axis, buoyant copper salt solution rises as a cylindrical jet of radius $R$. Based on our assumption, this is a classic hydrodynamical problem. The cylindrically symmetric velocity fields $v(r)$, which solve the Navier-Stokes equations, are

$$
v_{\mathrm{o}}=A_{\mathrm{o}} \ln r+B_{\mathrm{o}}+C_{\mathrm{o}} r^{2}, \quad v_{\mathrm{i}}=B_{\mathrm{i}}+C_{\mathrm{i}} r^{2},
$$

where we use indices "o" and " $\mathrm{i}$ " for the outer and inner fluid, respectively. Note that there is no logarithmic term in the expression for $v_{\mathrm{i}}$ because the velocity in the jet cannot diverge. The five constants $A_{\mathrm{o}}, B_{\mathrm{i}, \mathrm{o}}$ and $C_{\mathrm{i}, \mathrm{o}}$ in eqs. (1) are all fixed by imposing the proper boundary conditions: i) stick boundary condition $v_{\mathrm{o}}\left(R_{\text {cyl }}\right)=0$ at the cell wall; ii) continuity of elocity, shear stress and pressure at the fluid interface at $R$; iii) no-mean-flow constraint $\int_{R}^{R_{\text {cyl }}} \mathrm{d} r r v_{\mathrm{o}}(r)=0$ for the outer fluid since the overall volume of the silicate solution is constant. Hence, for fixed $R$ and given viscosities $\eta_{\mathrm{i}, \mathrm{o}}$ and densities $\rho_{\mathrm{i}, \mathrm{o}}$, the flow fields are completely determined. From $v_{\mathrm{i}}(r)$, we can therefore derive the mass flow $Q\left(R, R_{\text {cyl }}, \eta_{\mathrm{i}}, \eta_{\mathrm{o}}, \rho_{\mathrm{i}}, \rho_{\mathrm{o}}\right)$ through the jet. Since the fluid properties and the cell diameter are known, this yields a unique relation between $Q$ and $R$.

The latter $Q-R$ relation can be written out algebraically, but a general expression is too cumbersome to yield much physical insight. However, in the relevant limit $R_{\text {cyl }} \gg R$, the expressions simplify [14] to

$$
Q=\frac{\pi \Delta \rho g}{8 \eta_{\mathrm{i}}} R^{4}\left[1+\frac{4 \eta_{\mathrm{i}}}{\eta_{\mathrm{o}}}\left(\ln \left(\frac{R_{\mathrm{cyl}}}{R}\right)-1\right)\right], \quad \Delta \rho=\rho_{\mathrm{o}}-\rho_{\mathrm{i}} .
$$

The first term, with the $Q \sim R^{4}$ behavior, is simply the expression for Poiseuille flow through a pipe. The second term between square brackets is a correction factor that accounts for jetdriven motion in the outer fluid. In the case of a rigid tube, this term equals one. However, as we shall see, the data confirm that this induced flow is present, and that the weak-tube model is appropriate.

The result of the full calculation based on eq. (1) and the constraint of no-mean-outer flow is shown as the solid black line in fig. 2b. Notice that the underlying calculation involves no adjustable parameters but only quantities that are measurable in independent experiments and/or are reported in the literature [15-17]. These parameters along with the linearized concentration dependencies of $\eta_{\mathrm{i}}$ and $\rho_{\mathrm{i}}$ are specified under [18]. For this analysis, we find good agreement with the experimental results obtained for tubes grown in the jetting mode. The result of the approximation (eq. (2)) is plotted as a dashed line in fig. $2 \mathrm{~b}$ and is essentially identical to the original model. However, Poiseuille flow without the correction term (eq. (2) for $\eta_{\mathrm{o}} \rightarrow \infty$ ) is much steeper and does not agree with the data (cf. upper dashed curve in fig. 2). Moreover, none of the models shows satisfactory agreement for popping tubes. The fact that the corrected Poiseuille flow describes our measured data in the jetting regime so accurately justifies our starting assumption, i.e., the fact that the newly formed membrane in the growth region is thin and weak, so that the growth dynamics of the silica tube are dominated by hydrodynamics.

Figure 3 shows the outer radius of silica tubes as a function of seed concentration $\left[\mathrm{CuSO}_{4}\right]$. Our experiments reveal that for concentrations lower than $0.02 \mathrm{~mol} / \mathrm{L}$ no tubes are formed. The transition from jetting to popping dynamics occurs at $0.1 \mathrm{~mol} / \mathrm{L}$ (dashed lines in fig. 3 ) and budding growth is observed above $0.4 \mathrm{~mol} / \mathrm{L}$. We note that these transition concentrations show no pronounced dependence on flow rates for the range of parameters investigated. The 

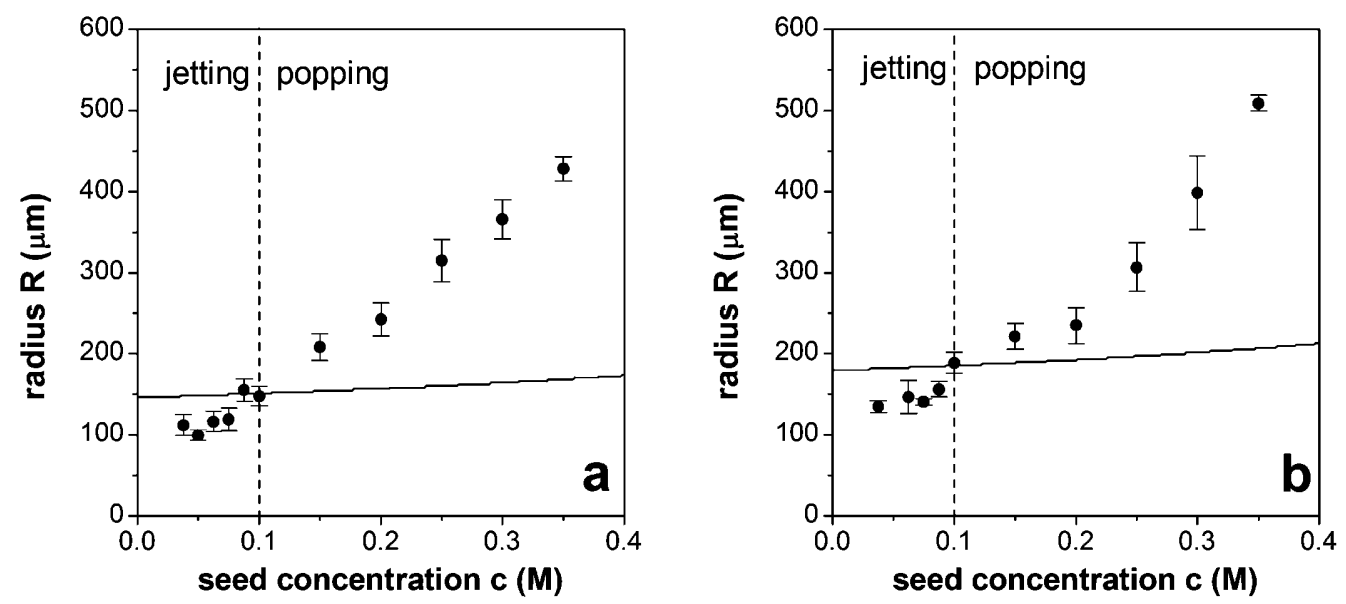

Fig. 3 - Concentration dependence of the outer radius of silica tubes. The flow rate is kept constant at 7.0 (a) and 15.0 (b) mL/h. The solid lines are calculated from a purely hydrodynamic analysis (cf. eq. (1)) using the given flow rates and concentration-dependent densities and viscosities. The dashed lines indicate the transition from jetting to popping growth.

two plots in fig. 3 are obtained for flow rates of $Q=7 \mathrm{~mL} / \mathrm{h}(\mathrm{a})$ and $15 \mathrm{~mL} / \mathrm{h}$ (b). The solid lines correspond to the concentration-dependent radii predicted by our hydrodynamic analysis based on eq. (1) and the parameter values/functions described above. In both cases, the experimental data for jetting tubes show a very weak dependence on seed concentration. For popping tubes, however, the radius increases rapidly with concentration. Our hydrodynamic model shows good agreement with the jetting data but does not recover the more pronounced dependence found for popping tubes. Although the model successfully captures the main features of the jetting data shown in figs. 2 and 3, it mildly overestimates the radius of the tubes. This is in agreement with our experimental observations indicating that the jets are slightly larger than the forming silica tubes (see, e.g., fig. 1a). Furthermore, our model possibly underestimates the flow rate $Q$ due to finite-size effects and an appreciable Reynolds number in the tube for large flow rates.

The above findings emphasize that the radii of popping tubes are not determined by buoyant jets of solution. In the following, we demonstrate that for this growth regime the loss of seed solution into the surrounding silicate medium is minimal. Figure 4a-h presents a sequence of micrographs of the top portion of a popping silica tube. The first row (a-d) illustrates the expansion of the membrane-bound droplet at the tip of the tube. As the droplet increases in volume due to the inflow of seed solution, the buoyant forces acting on the structure become stronger and give rise to rapid detachment of the droplet (c, d). During this phase, the tube is temporarily uncapped and solution is ejected in a very short $(\approx 0.1 \mathrm{~s})$ burst. However, the system undergoes fast reactions within the mixing zone of the two reactant solutions that induce the formation of a thin membrane (e,f). This membrane cap has a peculiarly pointed shape that seems to be the result of the fluid flow induced by the sudden burst of seed solution and/or the vertically departing droplet. Upon formation of the membrane, a new droplet forms that continues to expand (f-h) until buoyant forces are sufficiently strong to induce the next droplet detachment. The tube length increases during this cyclic process with a parameter-dependent, average speed of about $50-500 \mu \mathrm{m} / \mathrm{s}$ (for details see ref. [13]). This primary growth is dominated by silica precipitation that also causes a color change of 

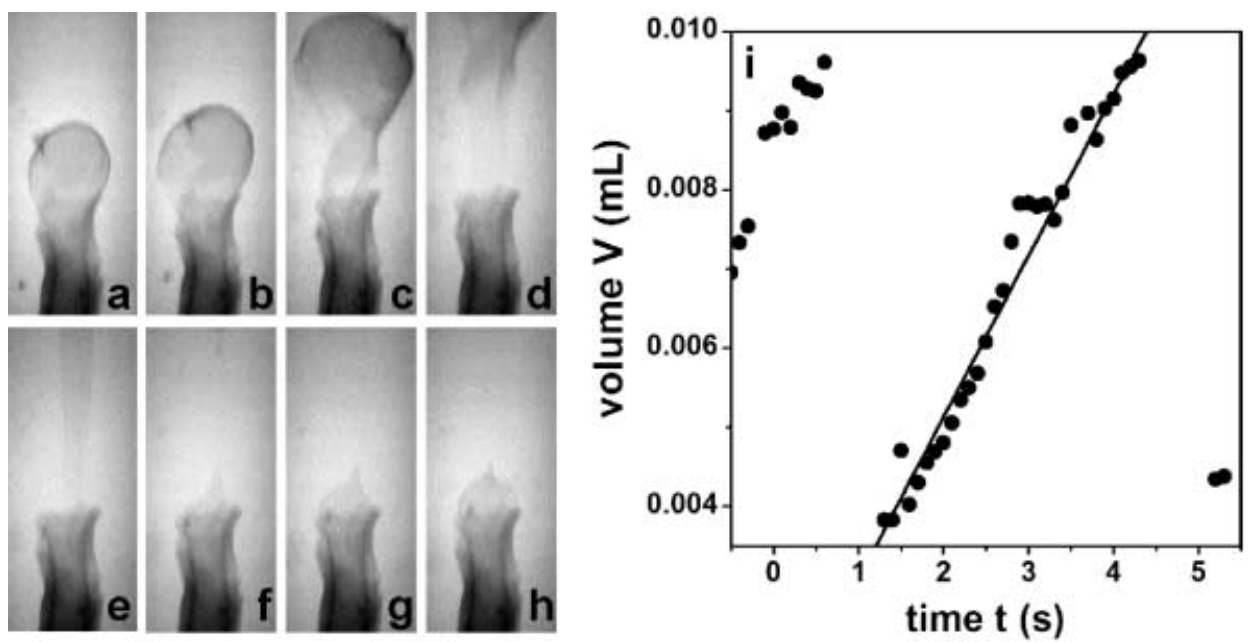

Fig. 4 - (a-h) Sequence of snapshots illustrating the expansion and detachment of membrane-bound droplets. Time of snapshots: $0.0,0.8,1.9,2.0,2.1,2.2,2.3,2.4 \mathrm{~s}$. Field of view: $2.8 \times 6.7 \mathrm{~mm}^{2}$. (i) Tube volume as a function of time (circles) with a superposed linear fit. The seed solution has a concentration of $\left[\mathrm{CuSO}_{4}\right]=0.3 \mathrm{~mol} / \mathrm{L}$ and is injected at $7.0 \mathrm{~mL} / \mathrm{h}$.

the transparent, faint blue top portion to the non-transparent, dark blue of the tube's main stalk. In this respect, the colloidal copper hydroxide membrane at the base of the droplet seems to be a scaffold for the slower silica precipitation at the membrane's outer surface.

To test the equality of the expansion rate of the droplets and the injection rate, we analyze video frames similar to those in fig. 4a-h. Our analysis is based on two steps that involve the automatic detection of the structure's outline and its volume reconstruction via Pappus's second theorem. This simple relation states that the volume $V$ of a body generated by the revolution of a lamina about an axis is equal to the product of the area $A$ of the lamina and the distance $d$ travelled by the lamina's centroid. With $\bar{x}$ denoting the distance of the latter point from the rotation axis, we obtain $V=A d=2 \pi A \bar{x}$.

In the following, we assume that the tube structure is a perfect body of revolution and that its symmetry axis is oriented in the vertical direction. From the structure's outline, we locate the position of the axis and compute the quantities $A$ and $\bar{x}$ of the corresponding left and right lamina. This procedure is repeated for all image frames of a given sequence and data obtained from the left and right laminae are averaged. Figure 4i shows a representative example for the temporal evolution of the volume of a popping silica tube. The graph has a sawtooth-like shape and involves one long phase of linear volume expansion that is followed by a rapid volume decrease due to droplet detachment. Assuming a linear dependence, we find that the rate of volume expansion equals $(7.3 \pm 0.5) \mathrm{mL} / \mathrm{h}$. This result is in excellent agreement with the pump rate of $7.0 \mathrm{~mL} / \mathrm{h}$ employed in this experiment. Hence, we conclude that the membrane-bound droplets of popping silica tubes show essentially no leakage of seed solution during the expansion phase.

In conclusion, our experiments show that the outer radius of silica tubes increases with increasing flow rates and concentrations of the seed solution. Although the corresponding data (see fig. 3) show no pronounced discontinuities at the transition from steady jetting to oscillatory popping growth, radius selection is governed by qualitatively different mechanisms. In the jetting mode, the tube radius is clearly determined by buoyancy-driven Poiseuille flow 
corrected for the presence of fluid motion in the surrounding silicate solution. Tube radii in the popping mode, however, show a $Q \propto R^{5.5 \pm 0.6}$-dependence which might result from the interplay of the internal flow within the silica tube and the buoyant force of the droplet: the buoyant pressure drop is proportional to the height of the droplet, which suggests $\Delta p \propto R$, and must be balanced by the viscous Poiseuille pressure, $\Delta p \propto Q / R^{4}$, in the tube. This dimensional analysis suggests a resulting mass flow of $Q \propto R^{5}$ that is consistent with the experimentally measured exponent of five to six. Lastly, we emphasize that our study provides the first quantitative analysis of the radii of self-organized tubular structures and its results could be applicable to various other precipitation systems [1-5].

$$
* * *
$$

This work was supported by the National Science Foundation (NSF CHE-20023105) and the American Chemical Society Petroleum Research Fund (ACS PRF 37278-G5).

\section{REFERENCES}

[1] Ackermann A., Kolloid Z., 59 (1932) 49.

[2] Fontana M. G., Corrosion Engineering (McGraw-Hill, New York) 1986.

[3] Double D. D., Hellawell A. and Perry S. J., Proc. R. Soc. London, Ser. A, 359 (1978) 435.

[4] Webster M., Educ. Chem., 35 (1998) 126.

[5] Cartwright J. H. E., Garcia-Ruiz J. M., Novella M. L. and Otálora F., J. Colloid Interface Sci., 256 (2002) 351.

[6] Maselko J., Geldenhuys A., Miller J. and Atwood D., Chem. Phys. Lett., 373 (2003) 563.

[7] Balköse D. et al., J. Sol-Gel Sci. Technol., 23 (2002) 253.

[8] Glauber J. R., Furni Novi Philosophici (Amsterdam) 1646.

[9] KeIr J., Philos. Trans., 80 (1790) 359.

[10] LeDuc S., The Mechanism of Life (Rebman, London) 1911.

[11] Collins C., Zhou W., Mackay A. L. and Klinowski J., Chem. Phys. Lett., 286 (1998) 88.

[12] Collins C. et al., Phys. Chem. Chem. Phys., 1 (1999) 3685.

[13] Thouvenel-Romans S. and Steinbock O., J. Am. Chem. Soc., 125 (2003) 4338.

[14] In an infinitely long cylinder, eq. (2) is based on the exact solution of the hydrodynamic equations for a laminar velocity field $v_{\mathrm{z}}(r)$. Just as in the Oseen discussion of laminar flow past a sphere, the effective Reynolds number at large distances increases for sufficiently large $R_{\text {cyl }}$. In reality, therefore, $Q$ does not diverge as $R_{\text {cyl }} \rightarrow \infty$, as eq. (2) appears to suggest at first sight.

[15] Gmelins Handbuch der anorganischen Chemie, Vol. 60, 8th edition (Verlag Chemie, Weinheim) 1958 , p. 531.

[16] Gmelins Handbuch der anorganischen Chemie, Vol. 21, 8th edition (Verlag Chemie, Weinheim) 1967, p. 1526.

[17] Roth W. A. and Scheel K. (Editors), Landolt-Börnstein - Physikalisch-chemische Tabellen, 5th edition, Vol. 1 (Edwards Brothers, Ann Arbor, MI) 1943, p. 136.

[18] The following data are used: $\eta_{\mathrm{i}} / \eta_{\mathrm{H}_{2} \mathrm{O}}=1.0+(0.7 \mathrm{~L} / \mathrm{mol}) c$ and $\rho_{\mathrm{i}}=\rho_{\mathrm{H}_{2} \mathrm{O}}+(0.15 \mathrm{~kg} / \mathrm{mol}) c$, where $c$ denotes the concentration of cupric sulfate. At $25^{\circ} \mathrm{C}$, the density and the viscosity of water equal $\eta_{\mathrm{H}_{2} \mathrm{O}}=0.895 \mathrm{cP}$ and $\rho_{\mathrm{H}_{2} \mathrm{O}}=997 \mathrm{~kg} / \mathrm{m}^{3}$, respectively. The silicate solution has a viscosity of $\eta_{\mathrm{o}}=1.74 \mathrm{cP}$ and a density of $\rho_{\mathrm{o}}=1124 \mathrm{~kg} / \mathrm{m}^{3}$. Accordingly, we find that $\Delta \rho=127 \mathrm{~kg} / \mathrm{m}^{3}-$ $(0.15 \mathrm{~kg} / \mathrm{mol}) c$. 